\title{
Health research priority setting in 0 man: Towards better utilization of the available resources
}

\author{
Adhra Al-Mawali*1,2 ,Ayaman Al-Harrasi**1, Sathish Kumar Jayapal 1 , Hilal Al-Kharusi ${ }^{1}$, Mariam Al-Rashdi 1, \\ Avinash Daniel Pinto'
}

\author{
'Centre of Studies \& Research, Ministry of Health, Sultanate of Oman \\ ${ }^{2}$ The Research Council. Sultanate of Oman \\ *First Author: Adhra Al-Mawali \\ **First Author: Ayaman Al Harrasi \\ Correspondence to Adhra Al-Mawali (adhra.almawali@moh.gov.om) \\ (Submitted: 03 May 2020 - Revised version received: 14 May 2020 - Accepted:28 May2020 - Published online: 26 June 2020)
}

\begin{abstract}
Objective This concept paper aims to portray the steps and strategies followed in setting the health research priorities in Sultanate of Oman. Methods This exercise was developed based on Council on Health Research for Development management process to priority setting for planning a high-quality health research priority setting exercise at national and subnational levels. Ranking for the diseases and risk factors in Oman was based on the estimates obtained from the Global Burden of Disease 2016 study by the Institute for Health Metrics and Evaluation. The most important topics were generated from a panel of experts to tackle the above-ranked problems which was supported with a feasibility score to plan for conducting research.

Results A list of top 30 diseases and 10 risk factors based on disability-adjusted life years estimates was ranked and listed. Subresearch topics were listed under each category. Feasibility scores were obtained from all the subnational levels based on available human resources, infrastructure, financing, technology, legality, and ethical consideration. A plan for the next 15 years was developed for each subnational level based on the feasibility score.

Conclusion Prioritization mechanisms are necessary to facilitate the current demand for increased harmonization of health research to meet health services needs, particularly in combination with translating the results into actions that improve overall population health. Due to the immense importance of health research in improving the health system and health services, the decision to prioritize health research was the first step in the direction to enhance health research and thereby enhance health service in Oman by the Centre of Studies \& Research. This intervention will lead the health system to achieve better planning for effective utilization of available resources.

Keywords Oman, Health research, Priorities, Health research priorities, Health research priority setting, COHRED
\end{abstract}

\section{Introduction}

It is well-known that better health for a greater portion of population leads to higher productivity and more equitable economic development. In the health sector, the benefits of implementing policies derived from sound evidence-based research may often yield desirable outcomes. Focusing on essential research leads to better use of available resources for health and ultimately to more health gains per dollar spent. In fact, one of the basic tenets of economics is that resources are always limited and there can never be enough of it to meet all the perceived needs. There will never be enough resources to address all the peoples' health needs, especially in developing countries. In resource rich settings, a high proportion of available research funds go to investigator-driven initiatives, but in limited resource settings, there is an expectation that research must respond more directly to community health needs, and therefore be conducted according to recognized priorities The outcomes and benefits cannot always be evaluated in economic terms. Hence, prioritizing domains for research has to be evaluated on several factors including burden of illness, impact on specific population groups, especially vulnerable sections of the society, health promotion, disease prevention, rehabilitation, societal impact etc.

Priority setting is increasingly recognized as essential to deliver widespread population health changes that respond to critical needs and contribute to sustainable developmental outcomes. Numerous World Health Assembly resolutions have stressed the need for action on prioritizing the health research activities for the following reasons:

- Health research prioritization is regarded as a key part of efforts needed to strengthen national health research systems.

- Most importantly, priority setting can provide valuable direction for the allocation of public and private research funds into areas of strategic importance. ${ }^{1}$

- Setting priorities for health research is essential to maximize the impact, which is especially relevant in resourcescarce environments.

- Setting priorities in research can serve to act as a catalyst for public debate, for bringing together different stakeholders and creating networks. These networks would ideally comprise researchers in the public and private sectors, decision-makers in governments, and civil society.

- Prioritization mechanisms are necessary to facilitate the current demand for increased harmonization of health research at a global level particularly in combination with analyses of financial flows for health research and burden of disease studies.

Due to the immense importance of health research in improving the health system and health service, the decision to prioritize health research was the first step in the direction to enhance health research, and thereby enhance health service in Oman by the Centre of Studies \& Research. This concept paper aims to portray the steps and strategies followed in setting the health research priorities in Sultanate of Oman in 2019. 


\section{Why Second Edition?}

The first edition of health research priorities in Oman was published in April 2014. Research domains were identified based on Delphi method, considering the priorities of research emanating from the eighth 5-year plan for the development of health, regional research priorities, research priorities recommended by international organizations and research priorities that serve the health vision for 2050 .

As we move towards 2050, these domains have to be reviewed and updated periodically in the light of the changing spectrum of health problems. In Oman between 1970 and 1990, communicable diseases were a major health problem. Through active intervention under wise leadership, considerable progress has been achieved and most preventable communicable diseases have been almost eliminated. By the turn of this century, the burden of disease has transitioned to NonCommunicable Diseases and a few of the newer communicable diseases. ${ }^{2}$ It is easy to see why this dynamic process of periodic recasting of the priority in research has to be practiced, so that the focus remains on the important issues that can produce the most beneficial effects in health care and health delivery. What will be the health issues that require research by 2050 ? It will be difficult to predict now, but through a dynamic process of prioritizing health research it can be on track at all times and in changing situations.

\section{Materials and Methods}

The question of how priority setting processes work remains topical, contentious, and political in every health system across the globe. It is particularly acute in the context of developing countries because of the mismatch between needs and resources, which is often compounded by an underdeveloped capacity for researchers and weak institutional infrastructures for research. ${ }^{2}$ Yet, there is limited research into how the process of setting and implementing health research priorities works in developing countries.

In 1990, the commission on health research and development drew attention to the need for essential national health research for developing countries. The Commission on Health Research for Development advocated the use of a systematized approach to priority setting within each country's Essential National Health Research (ENHR) strategy. The Council on Health Research for Development (COHRED) was established to assist developing countries with the implementation of this strategy. ${ }^{4}$ COHRED emphasized the following principles:

- There is a need for solid evidence to underpin an inclusive health research agenda.

- There is a need to involve all stakeholders in the prioritization process.

- And there is a need to link research results to policy and action.

Based on COHRED's experience, there is no one best method for priority setting. They strongly suggest those responsible for priority setting to weigh complexity of methods against what is to be achieved and what resources are available.

\section{What is the Process of Research Priority Setting Followed in Oman?}

For health research priority setting exercises to effectively target research with the greatest public health benefit, it is important that they are of high quality. There are various approaches available to guide priority setting for health research which differ on important aspects of the process. Therefore, taking the heterogeneous nature of research priority setting exercises and the different contexts for which priorities can be set, the optimal approach varies per exercise. ${ }^{5}$ Consensus on a gold-standard or best practice for health research prioritization, thus seems difficult to achieve and is, more importantly, not an appropriate response.

But there are nine common themes of good practice for health research prioritization processes which have been used by the Centre of Studies and Research in Oman based on COHRED recommendation to provide assistance for planning a high-quality health research priority setting exercise at national and subnational levels. There are nine common themes of areas to focus during the exercise which are: ${ }^{6}$

1. Assessing the contextual factors

2. Choosing the approach

3. Assess the inclusiveness

4. Identify the information

5. Choosing the criteria

6. Adapt methods

7. Evaluation

8. Transparency

9. Planning for implementation.

\section{Assessing the Contextual Factors}

There are several contextual factors that underpin the process of research priority setting, namely practical considerations about available resources, the focus of the exercise, the values that stakeholders adhere to, and the health, research, economic, and political environment in a country. These factors influence the prioritization process and the eventual research priorities and should therefore be discussed explicitly from the beginning of the exercise. Careful planning of the prioritization exercise is important to establish an exercise that meets the initial expectations. It is necessary to identify available financial, human, and time resource. The contextual elements were determined as following:

- The information resources which were available for the exercise were:

- Institute for Health Metrics and Evaluation's Burden of Diseases 2016 study

- Annual Health Reports

- Health Research Priorities 2014 ( $1^{\text {st }}$ version)

- Health Vision 2050

- 5 years plan for health research (CSR)

- Experts' opinion

- Review of Literature

- The focus of the exercise:

- To identify the research priorities in diseases and their risk factors and health system.

- To ensure that the priorities are used by practicing health workers, students of health sciences, academicians or researchers from other institutions with common interest, and by stakeholders and decision-makers.

- Health, research, and the political environment in which the process will take place is detailed in the "Health Vision 2050 "and was revised thoroughly. ${ }^{7}$ Here are the main aspects related to the research prioritizing exercise at the time of health research priority setting (2016): 
- Health Status (2016): 8

- The health system in Oman is characterized by its universal health coverage for both citizens and nonnationals. Health care is directly provided in facilities mainly owned and operated by the Government. The Government provides about $79 \%$ of hospitals and about $91 \%$ of hospital beds. Public health services are run by $78 \%$ of doctors, $84 \%$ of nurses, and $78 \%$ of other paramedics. About $66 \%$ of dentists and $71 \%$ of pharmacists work in the private sector.

- The Ministry of Health (MoH) is the main healthcare provider and is responsible for ensuring the availability of health policies and plans and monitoring their implementations. Other health-care providers in the country include: Armed Forces Medical Services, Royal Oman Police Medical Services, Sultan Qaboos University Hospital, Diwan Medical Services, Petroleum Development Oman Medical Services, and the Private Sector.

- The Omani health system is a free-medical care health system, chiefly financed through Government revenues. The Government is committed to providing health care and services to all citizens free of charge and has considered equity in financing health services across different health Governorates with the aim of ensuring financial protection for all. ${ }^{9}$ NonOmani residents receive their medical care mainly in private health-care facilities

- MoH provides health care mainly to Omani citizens through 49 hospitals and 205 health centers scattered across the country. $\mathrm{MoH}$ distributed hospitals across all Governorates, such that each Governorate is served by a Governorate Hospital (GH) (10) aided by a Wilayat Hospital (WH) (5) in some populated Governorates to provide secondary care to their inhabitants (secondary health care is also provided by two extended health centers and one local hospital)

- These secondary care hospitals are apexed by four national referral hospitals (NRH) (located in Muscat Governorate) that provide tertiary care to citizens of Oman. $\mathrm{MoH}$ provides primary health care through Health Centers (182 HCs), Extended Health Centers (21 EHCs) and local hospitals (29 LH) distributed across all Governorates.

- A total of 6,393 physicians, 14,675 nurses, 358 dentists, 554 pharmacists, and 6,234 paramedical staff in addition to 12,050 medical orderlies and support staff run health services in $\mathrm{MoH}$ health-care facilities (2016).

- Political and Economic Status (2016):

- It is important to scan the political situation in Oman to show the geopolitical stability supporting different aspects of development including health. Oman was one of the least known countries and remained largely isolated from the rest of the world until 1970 when His Majesty Sultan Qaboos bin Said came to power. His Majesty's reign signaled the Renaissance or the beginning of a bright new era that renewed Oman's historic past and opened a new chapter of development, prosperity and social and economic progress.
- Oman is currently described as a high-income country. It is a relatively large country with an area of about $309,500 \mathrm{~km}$. It has difficult terrain and an intricate topography, with high and rugged mountains and barren valleys. Its small population of 4.2 million, of which $43.6 \%$ are expatriates or nonnationals, is scattered over large areas of sparsely populate settlements.

- The Sultanate of Oman evolved to become a modern country with state-of-the-art services under the rule of Sultan Qaboos, which began in 1970. In 2015, its gross domestic product (GDP) at current prices has grown to Omani Rials (OMR) 26,850.3 million (US69,922.7 million). The graph below showing the expenditure on health in Oman from 1995 to 2015.

- Administratively, the country is divided into 11 governorates with 61 wilayats (districts). Each governorate is considered a health region. These governorates are: Muscat, Dhofar, Musandam, Al-Buraimi, Ad Dakhiliyah, North Batinah, South Batinah, South Sharqiyah, North Sharqiyah, Al-Dhahirah, and Al-Wusta.

- Leadership and governance in the Omani health system has responsibly and wisely managed resources and revenues to the benefit of the health of the people of Oman and has responded to their needs during its different stages of development. Sound policies, strategies, and development plans have been adopted. The Government of Oman, through the Ministry of Health, has a health policy that is based upon several basic principles: Provision of comprehensive public and personal health services to its population through a health system with primary health care as its cornerstone. Equity in the distribution of health services and fairness of financial contribution among different population groups according to health needs. Community involvement in planning and implementation of its health care aimed at developing community self-reliance for sustainable health development, responsiveness to health and nonhealth needs of the community and Intersectoral cooperation with other health-related sectors to ensure positive impact on community health. ${ }^{10}$

- The Consultative Council: In November 1991, Sultan Qaboos replaced the 10-year-old State Consultative Council with the Consultative Assembly (Majlis al-Shura) to systematize and broaden public participation in government. The Assembly has 84 elected members and exercise some legislative powers. Representatives were chosen in the following manner: Local caucuses in each of the 59 districts sent forward the names of 3 nominees, whose credentials were reviewed by a cabinet committee. These names were then forwarded to the Sultan, who made the final selection. The Consultative Assembly serves as an information channel between the people and the government ministries. It is empowered to review drafts of economic and social legislation prepared by service ministries, such as communications and housing, and to provide recommendations. Service ministers also may be summoned before the Majlis 


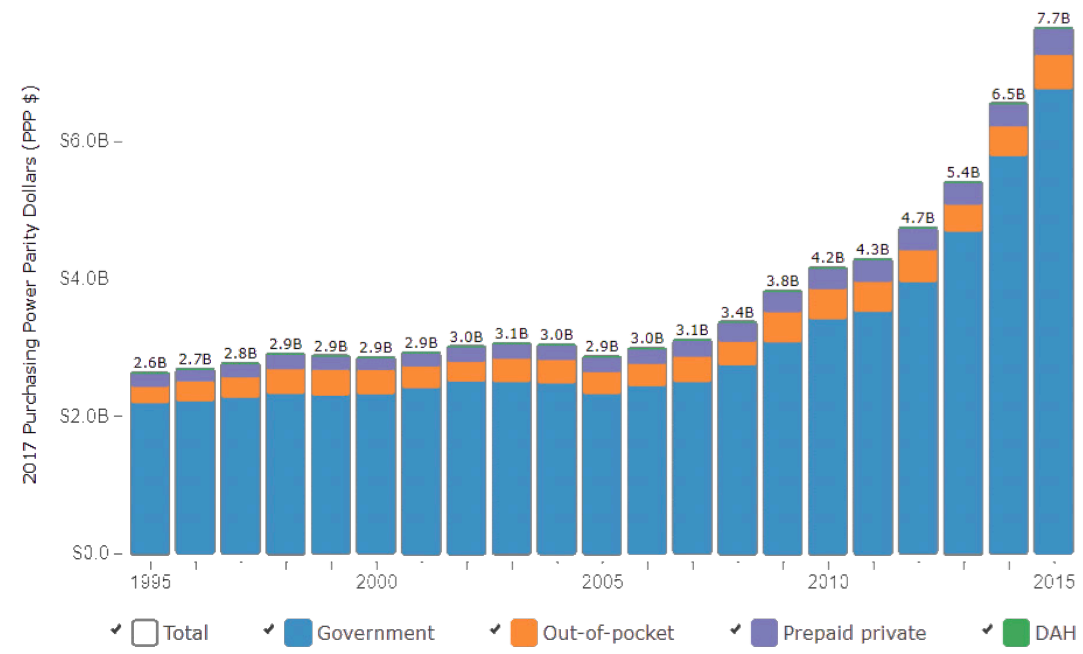

Fig. 1 Expenditure on health in Oman from 1995 to 2015.

to respond to representatives' questions. It has no authority in the areas of foreign affairs, defence, security, and finances. The Council of State (Majlis al-Dawla) has 83 appointed members including 14 women.

- Research status

- The Centre of Studies \& Research (CSR) is the official body and focal point within the Ministry of Health which is responsible for implementing the policies of the Ministry of Health for promoting research culture and governing research activities in the health sector in Oman. It has been providing evidence-based information to decision makers and other researchers/academicians. ${ }^{11}$

- The Research Council Oman (TRC) was established on June 22, 2005, pursuant to the Royal Decree number 54/2005. It carries tasks related to research and endeavors to promote and support research using all the material and moral means possible. The Research Council in its capacity is the main national authority in this area, acts as a focal point for research and innovation and liaise with various institutions concerned with research. The Research Council is both a policy-making institution and a funding agency. It is offering funding called Block Funding Program (BFP) which is an institutional- and performance-based funding. This program allocates small-to-medium size research grants to support short- and mid-term research projects in areas defined by researchers from academic and research institutions in Oman and serve the national research priority areas as well as TRC priority themes. The main goal of this program is to sustain and develop further excellent in research and to create a competitive economy through advanced and evidence -based research (Fig. 2).

- However, research in Oman is still considered of limited benefits. The SCImago Journal and Country Rank portal assessed countries for the number and citations of publications during 1996-2010. It showed that Oman had only 1,522 publications with
7,357 citations. This was not considered favorable and Oman was ranked ninth among 15 countries in the Middle East and North Africa region. A study published in 2011 showed that more than one-quarter of biomedical publications by Omani researchers during the period 2005-2009 were published in journals with no Impact Factor (IF) and more than half were in journals having IF of less than 1 . The study concluded that the quality of research originating from Oman is of limited usefulness.

- Almost all of the health-care facilities run by $\mathrm{MoH}$ and other public health-care providers are fully computerized. Patients "records are managed electronically, and a wealth of patient information is available. The situation is not the same in the private sector. In spite of the fact that $\mathrm{MoH}$ hospitals and primary health-care units are fully computerized, data are not directly extracted from health institutions" databases by the NHSIS. Coordination between NHSIS and IT is a challenge for sustaining the flow of health data and information. The absence of a countrywide electronic connectivity of all health-care facilities is another challenge for enhanced health service delivery and for extracting health data.
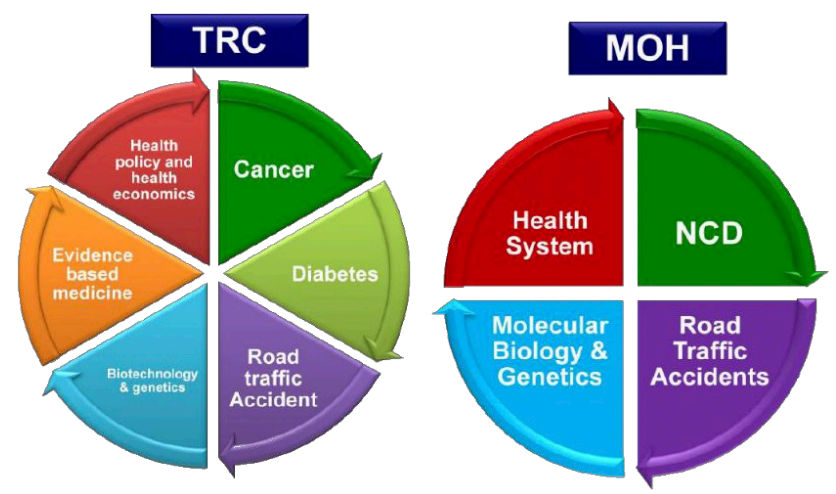

Fig. 2 Health Research bodies in 0 man and the types of health research they covered. 
- There are a number of challenges from enhancing and developing health research; these would include:

- The lack of sufficient and allocated funds for health research in Ministry of Health.

- Non-adherence of the academia and national programs to the identified research priorities of the $\mathrm{MoH}$.

- Weak co-ordination of research activities between the $\mathrm{MoH}$ and academia-supported researchers within and outside the country and poor communication of the research results.

- A limited number of identified research topics in the health development plans are implemented.

- Lack of close monitoring of research activities and their outcome.

- A "Research culture" has not permeated sufficiently among health care professionals.

- Although collaboration with reputed institutions in other countries exists in the field of health-care services and academia, collaborative research is minimal.

- Health service in Oman is highly reliant on medical products originating in and based on research conducted in other countries.

- Private donor agencies are motivated to grant donations for health care but not for research due to lack of sufficient awareness of the relevance of health research.

- Infrastructure for conducting innovative research, particularly in the area of medical products and technology, is lacking.

- Lack of sufficient experience for research in congenital anomalies and genetic disorders is an important challenge to the health system in Oman.

- Poor access to the benefits and products of research, despite dramatic advances in knowledge and technology.

\section{Choosing the Approach Used to Health Research Priorities Setting}

There are number of comprehensive approaches to health research priority setting. These approaches are comprehensive because they provide structured, detailed, step-by-step guidance for the entire priority setting process, covering many of the points on this checklist. They assist in the preparatory work of an exercise, in deciding on priorities, and in what to do after priorities have been set. Use of these approaches is therefore in general advantageous and their use should be at least considered. The four commonly used comprehensive approaches are:

1. 3D Combined Approach Matrix (CAM).14

2. Essential National Health Research (ENHR) approach.

3. The Child Health and Nutrition Research Initiative (CHNRI) approach.

4. The COHRED management process to priority setting.

The one adapted in Oman's exercise of priority setting is COHRED (Commission On Health Research For Development) as it focuses on the management process for national-level exercises. This high-level approach delineates important steps of a priority setting process for national-level exercises and discusses a wide range of options for tools and approaches to use in the process.

Over the past 15 years, COHRED has supported countries in setting national priorities for health research. Based on this experience, COHRED has developed an integrative approach that countries can use to manage their priority setting process. The present approach has been structured as a comprehensive guide that will help the users in designing the most appropriate priority setting process for their countries. To facilitate action practical ideas, management tools, existing priority setting methods and techniques, reference documents and country examples are proposed. The approach reveals priority setting as a cyclic management process where six key practical steps are identified:

1. Assessing the situation.

2. Setting the scene.

3. Choosing the best method.

4. Planning priority setting.

5. Setting priorities.

6. Making priorities work.

COHRED is an international nongovernmental organization whose primary objective is to strengthen research for health and innovation systems, with a focus on low- and middle-income countries. COHRED supports countries to use research for health and innovation to: Improve health and reduce health inequities. This guide of COHRED is meant for any country, region or institution that wants to make a difference in health, equity and development through research. ${ }^{15}$ They recommend the following:

- Research priorities should be credibly set and regularly updated: set a date for an update already at the start.

- Ensure the process is inclusive. This is as important as the methodology used to define priorities.

- COHRED suggest not to allocate resources to the defined priorities at once. Allow some financial flexibility for innovation, blue sky research or unexpected health challenges and opportunities

\section{Assess the Inclusiveness of Health Research Priorities Research Setting Process}

In principle, broad stakeholder involvement (multisectoral and multidisciplinary) is beneficial for the outcomes of a research priority setting exercise for several reasons. Firstly, it minimizes the chances of research options being overlooked. Different groups of stakeholders tend to prioritize research differently. Secondly, participation in the exercise fosters ownership of the established priorities among those involved, thus increasing the chances of implementation of the priorities. Thirdly, broad participation makes priorities correspond to the needs of those that will implement and those that will benefit from the research priorities. As such, the prioritized research will be a better response to societal and policy needs, increasing the overall credibility of the exercise and the potential impact on health and health equity. Finally, broad stakeholder involvement may prevent unnecessary duplication of prioritization efforts and hence wasting of resources. Lastly, appropriate leadership of the priority setting process needs to be identified. This can for example be in the form of an executive committee or an advisory group that provides overall guidance on the prioritization process, while a 
larger core working group or decision-making group actually decides on priorities. Good leadership can be pivotal in creating and sustaining a high-quality priority setting process. In second edition of health research priority setting, there was appropriate representation of expertise and balanced gender and regional participation. All elements of health sectors have been included.

\section{Identify the Information Needed to Be Gathered for Health Research Priorities Setting}

There are many ways to make the priority setting process better informed and choices should be made on which types of information are necessary. These can include the collection of technical data that are often needed to inform discussion on research priorities (See Criteria), such as burden of disease, cost-effectiveness of interventions, current resource flows towards particular research areas, or determinants of disease. Furthermore, in order to be able to prioritize research, one must first know where the gaps in knowledge are; a literature review to identify those gaps is often necessary. ${ }^{16}$ Also, an initial survey of broader stakeholder views on priorities or opinions on matters related to the research area, or a review or impact analysis of previously established priorities can serve as preparation before the actual exercise. Furthermore, research priority setting is needed at different geographical levels: national, local within governorates, and within wilayats. For most health research topics, priorities will be the same on all levels. For most however, priorities will reflect the context they are seeking to address. The information gathered to conduct the exercise were:

1. Literature reviews on how to conduct the exercise of priority setting.

2. Collection of technical data (e.g., burden of disease, mortality, morbidity).

3. Assessment of broader stakeholder views.

4. Expert opinions.

5. Reviews or impact analyses of previous priority setting exercises and exercises from other geographical levels.

\section{Choosing the Criteria for Health Research Priorities Setting}

Commonly, criteria can be categorized into one of three dimensions: Public health benefit (should we do it?), feasibility (can we do it?) and cost. Participants in the priority setting exercise should decide by consensus on appropriate criteria at the beginning of the exercise. The following questions were answered as following to reach consensus on the criteria used by the Centre of Studies and Research to set the health research priorities:

\section{1 - (Should we do it?) Public health benefit}

In order to answer this question, the focus of the second edition of priority setting was to answer the following three questions:

\section{Magnitude of a health problem:}

In order to align health systems with the populations they serve, policymakers first need to understand the true nature of their country's health challenges - and how those challenges are shifting over time. That means more than just estimating disease prevalence, such as the number of people with depression or diabetes in a population. Global Burden of Disease (GBD) data incorporate both the prevalence of a given disease or risk factor and the relative harm it causes. The tools allow decision-makers to compare the effects of different diseases, such as malaria versus cancer, and then use that information at home. The disability-adjusted life year (DALY) is a measure of overall disease burden. The DALY metric is composed of years of life lost (YLL) due to disease causing mortality and years lived with disability (YLD). This provides high-quality epidemiological data on health status that are independent of interest groups. The health research priorities setting (2018) by CSR adapted the DALYs estimates from The Global Burden of Disease (GBD) 2016 Study by The Institute for Health Metrics and Evaluation (IHME). ${ }^{15,16}$

To make these estimates more accessible and useful, IHME has distilled large amounts of complicated information into a suite of interactive data visualizations that allow people to make sense of the over 1 billion data points generated.

Collected and analyzed by a consortium of more than 3,000 researchers in more than 130 countries, the data capture premature death and disability from more than 300 diseases and injuries in 195 countries, by age and sex, from 1990 to the present, allowing comparisons over time, across age groups, and among populations. The flexible design of the GBD machinery allows for regular updates as new data and epidemiological studies are made available. In that way, the tools can be used at the global, national, and local levels to understand health trends over time, just like gross domestic product data are used to monitor a country's economic activity. Policymakers in Brazil, China, India, Indonesia, Mexico, the United Kingdom, and other countries worldwide are collaborating with GBD researchers to adopt this approach for measuring their population's health and how it varies by different regions, socioeconomic status, or ethnic groups in their country.

The Global Burden of Disease (GBD) 2016 Study is a systematic assessment of the disability and mortality of major diseases and risk factors worldwide. It is a collaborative effort of scientists and researchers from the World Health Organization (WHO), World Bank, Institute for Health Metrics and Evaluation (IHME), Harvard School of Public Health, and University of Auckland School of Population Health. GBD 2016 estimates the burden of 291 diseases and injuries in 187 countries from 1990 to 2015.

Thus, list of 30 diseases and 10 risk factors where ranked based on DALYs estimates from the above-mentioned study. Thus, the ranking is independent of interest group.

\section{What About Research to Strengthen Health Systems}

This is particularly true when a systems perspective is used, i.e., by considering all the positive and negative effects of a particular system-level intervention, this research can provide a robust and accurate understanding of health systems challenges and their potential solutions, thereby improving the utility of the findings in other settings. This systems approach, in combination with stakeholder engagement, also informs the definition of priority research questions to 
address health systems challenges. Health systems research by necessity is highly multidisciplinary, with a strong emphasis on social sciences, economics, and anthropological investigations. Much ongoing research consists of descriptive, comparative, and evaluation studies and secondary analytical research. Although experimental studies are less common, partly because of operational and ethical challenges in experimenting at the health system level, they can be very informative and provide convincing evidence on the benefit of innovations in health system efficiency and health impact. The research in this domain falls under the general definition by the Alliance for Health Policy and Systems Research (HPSR) as: "The production of new knowledge to improve how societies organize themselves to achieve health goals." The Alliance for HPSR further clarifies that the prime focus of health policy and systems research is not a specific disease or service, but rather the health system as a whole. However, health systems research sometimes adopts a disease or service specific. More specifically, it can address any or all of the six building blocks of health systems identified by the WHO: service delivery, information and evidence, medical products and technologies, health workforce, health financing, and leadership and governance. In doing so, it should explicitly acknowledge the importance of the continuous interactions between the different building blocks of the health systems and the different sectors (including nonhealth sectors) involved, as well as all the other characteristics of complex health systems. Another definition refers to health systems research as "research that enhances the efficiency and effectiveness of the health system." Research on health systems addresses a huge research area that has only been marginally covered to date. Because of the multitude of system challenges and their complex multidimensional environment, research prioritization is essential, and some recent priority-setting initiatives are being timely. Due to the relative scarcity of research capacity to undertake this type of research, efforts to improve the design, robustness, and applicability of the evidence generated in one setting to another would be highly desirable. Systems thinking methods and approaches can offer tremendous help and guidance on this. By using a systematic, comprehensive way of examining the design and evaluation of potential health systems interventions, and ensuring involvement and ownership of all stakeholders involved, the utility and pay back from the evidence generated from this research greatly increases.

The Centre of Studies \& Research with collaboration with Health Policy and System department in Ministry of Health had categorized the research in health systems into two main categories (Fig. 6)

1. Research related to functions of health system. (input + process).

2. Research related to outcome of health system. (output).

\section{Likelihood of Reducing Disease Burden}

The list of diseases and risk factors and health system issues ranked by DALYs were assessed for the likelihood of reducing the burden. They all are having moderate to high likelihood for reducing their burden according to the experts.

\section{Present Level of Knowledge}

The lists were assessed for the present level of knowledge. It has been found that there is a need for more and accurate information in order to tackle them effectively. Thus, list of recommended topics where integrated with the above list of diseases and risk factors as can be seen in Table 1 .

\section{Feasibility (Can We Do It?)}

Instead of assessing the feasibility of the main research list of health problems and risk factors, topics or research questions of each health problem are assessed from the following six feasibility perspectives:

- Infrastructure (IS).

- Human resources/workforce (HR).

- Technology (T).

- Finance (Fin).

- Ethical aspects (Eth).

- Legality (L).

Each perspective will be scored from 0 to 2 denoting:

0 : not available / not currently legal / not currently approved

1: available but not sufficient /legal /ethically approved

2: sufficient

The maximum score is 10; and the higher the score, the higher feasibility the topic will have. The feasibility score can be used to categorize the specific research questions into short, intermediate and long-term list of specific research topics as following:

- Short-term priorities (to be done with 5 yrs.): feasibility score $=(7-10)$

- Intermediate-term priorities (to be done with $10 \mathrm{yrs}$.): feasibility score $=(4-6)$

- Long-term priorities (to be done with 5 yrs.): feasibility score $=(0-3)$.

The above feasibility analysis has been integrating into the list of the diseases and risk factors as in Table 1.

\section{Cost Effectiveness (Is It Cost Effective?)}

a) There is big gap of knowledge in this area which should be filled through building capacity.

b) Can be used in the next edition of research priorities exercise as a tool to rank health research priorities.

\section{Adapt Methods for Health Research Priorities Setting}

As COHRED is the approach adapted by the Centre of Studies \& Research, its recommendation is to use methods suited to local context and needs. As recommended by COHRED, the exercise of priorities setting should consider the use of more than one method to optimise the usefulness of results, and adapting methods to specific setting, available data and resources, and to local needs. ${ }^{1,22}$

In General, there are two main categories of methods for identifying the health issues:

- First category: using and compiling existing data (compound approaches) for example:

- Essential National Health Research

- Burden of Disease

- 3D Combined Approach Matrix ${ }^{23}$

- Child Health Priorities 


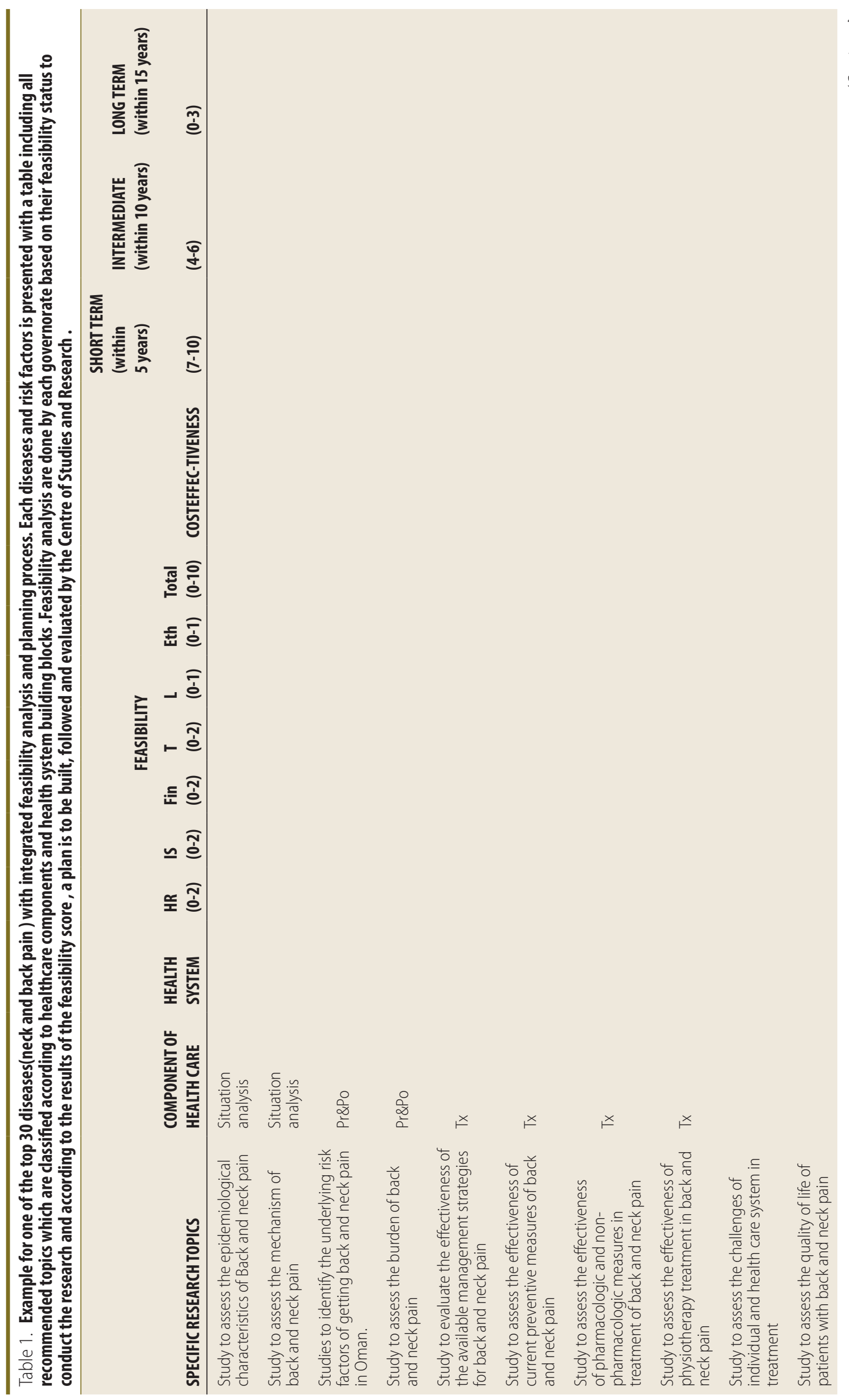



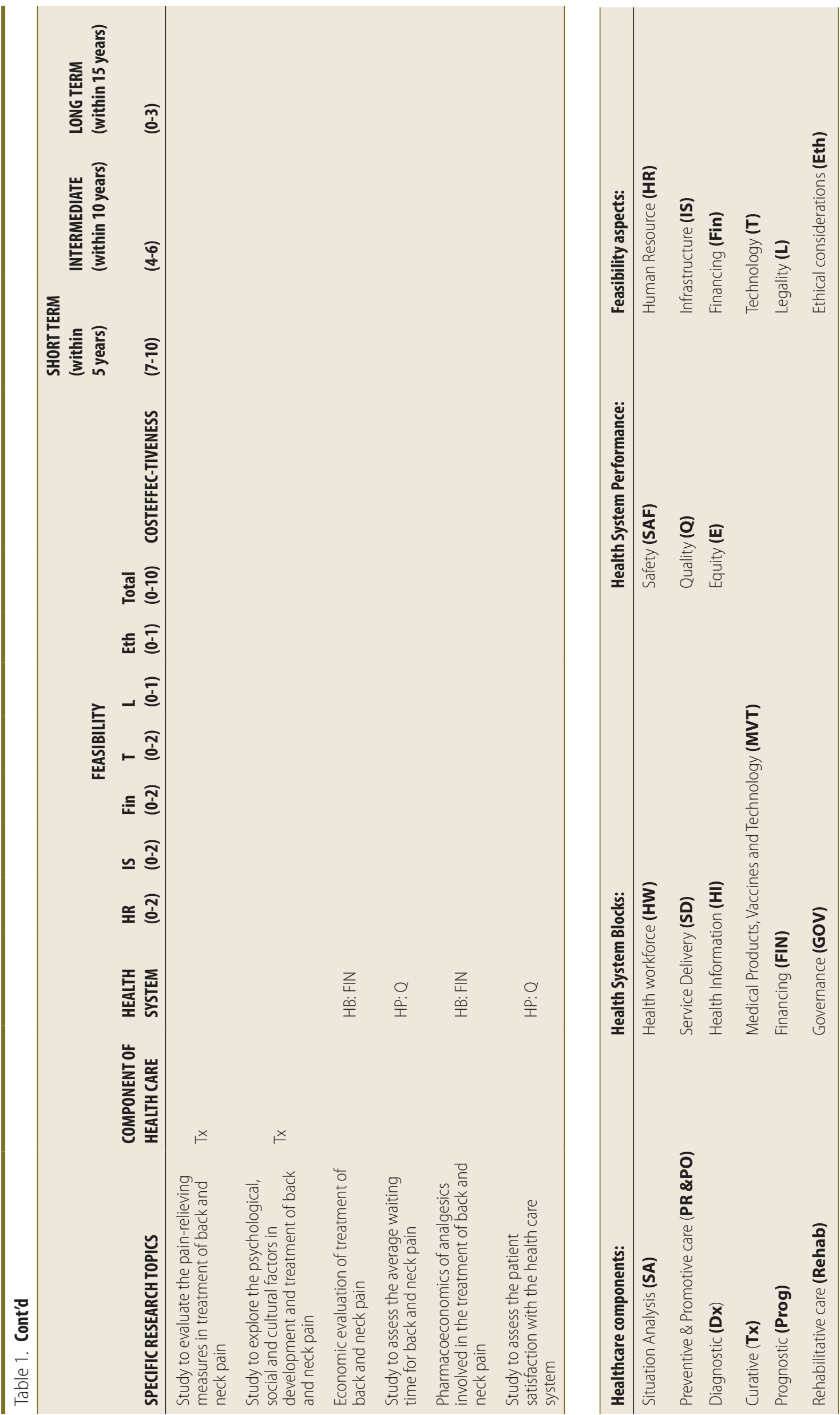
- Second category: providing insight in future health priorities (foresight techniques):

- Visioning

- Scenario creation

- Delphi

- Roadmaps

What is best for Oman in setting health research priorities?

In Oman, both methods are applicable, as we have reliable mortality and morbidity data for substantial parts of the population with IHME estimates of GBD (2016) as well as having" Health Vision 2050" which is considered to be the roadmap for the planning in the future. ${ }^{23,24,25}$ So, combining both methods for setting health research priority in Oman will give the most reliable list of priorities. ${ }^{26}$

\section{Evaluation}

The identification of health research priorities should be seen in the broader context of health research coordination and inform funding and policymaking for health research in a sustainable manner. ${ }^{27}$ Hence, previously set priorities should be periodically reviewed to ensure that priorities are up to date. Besides updating research priorities, other forms of evaluation can be considered. Evaluation of the process used to set priorities can increase the quality and acceptability of that process. Furthermore, to make research prioritization legitimate and fair, an appeals mechanism for the established priorities can be considered, providing opportunity for feedback. ${ }^{28}$ Finally, performing an impact analysis, for example in the form of a review of research performed and/or funding allocated based on previously established priorities, can be valuable. Not only can this provide insight into priorities that have remained devoid of attention, but it can also enforce discussion on implementation issues. The evaluation of the established priorities and the priority setting process will take place in periodic manner. Health research priority setting will not be a one-time exercise. ${ }^{29}$ The achievement of each governorate will be evaluated yearly and graded by star grading system:

- 1 star: achieving $20 \%$ of 5 year (short term) specific research questions list of the governorate.

- 2 stars: achieving $40 \%$ of 5 year (short term) specific research questions list of the governorate.

- 3 stars: achieving $60 \%$ of 5 year (short term) specific research questions list of the governorate.

- 4 stars: achieving $80 \%$ of 5 year (short term) specific research questions list of the governorate.

- 5 stars: achieving $100 \%$ of 5 year (short term) specific research questions list of the governorate.

\section{Transparency}

Documenting all steps followed in building the second edition of health research priorities by publishing a report, to be as transparent as possible is crucial. Potential implementers of health research priorities are unlikely to adopt or use priorities unless they are fully informed of all aspects of the priority setting process; transparency increases the credibility and thus the acceptability of the final result. ${ }^{30}$ Therefore, the report should not be limited to stating a list of priorities, but should also explain how those priorities were established, and by WHO. ${ }^{31}$ This entails providing details on which choices were made for points one through eight on this checklist, and why those choices were made. ${ }^{32}$ The documented was distributed through the official channels of Ministry of Health and workshops was documented at each governorate to ensure full understanding of the objectives of the prioritizing process.

\section{Planning for Implementation}

The plan for implementation is considered to be as follows: ${ }^{33}$

- All research regions will follow the national health research priorities for diseases, risk factors and the health system.

- Feasibility assessment of the specific research questions for each health issue or problem will be done by each research region to generate short-, intermediate- and long-term lists of specific health research questions or topics.

- The list of priorities to be announced in a ceremony in each research region with the presence of all community sectors (including private parties)

- Research groups for the short-term topic to be established within each research region.

- Recruitments of researchers for the research groups with the needed qualifications to be announced in the media channels including the webpage of each research region in the CSR website.

- Research Methodology Training programs to be linked with the research groups activities:

- Level one: Introduction + Basic concepts

- Level two: Advanced Research methodology skills

- Level three: Writing the proposal

- Research activities to be followed by a team from research section in each governorate.

- A report of achievements to be submitted to CSR at the end of each year.

- Achievements to be graded by star grading system.

- Grade of each research region to be announced in the webpage of each governorate in the CSR website.

- All research regions will compete every year to win the top best achievement in the 5 years short-term health research priorities plan.

\section{Results}

Following the above-mentioned methodology, a list of 30 diseases and 10 risk factors where ranked based on DALYs estimates from the Global burden of Diseases study.

\section{Top Risk Factors List}

In general, the risk factors can be categorized into Metabolic, behavioral and environmental/occupational risk factors. Globally, behavioral risks are causing the most disease burden, but in the Oman, metabolic risks are the most causative risks of the disease burden ${ }^{17}$ (Fig. 3).

In 2016, the risk factors that drive the most disease burden (DALYs) in Oman are as following:

1. High body-mass index (BMI)

2. High fasting plasma glucose

3. Dietary risks

4. High blood pressure

5. High total cholesterol

6. Occupational risks

7. Tobacco 


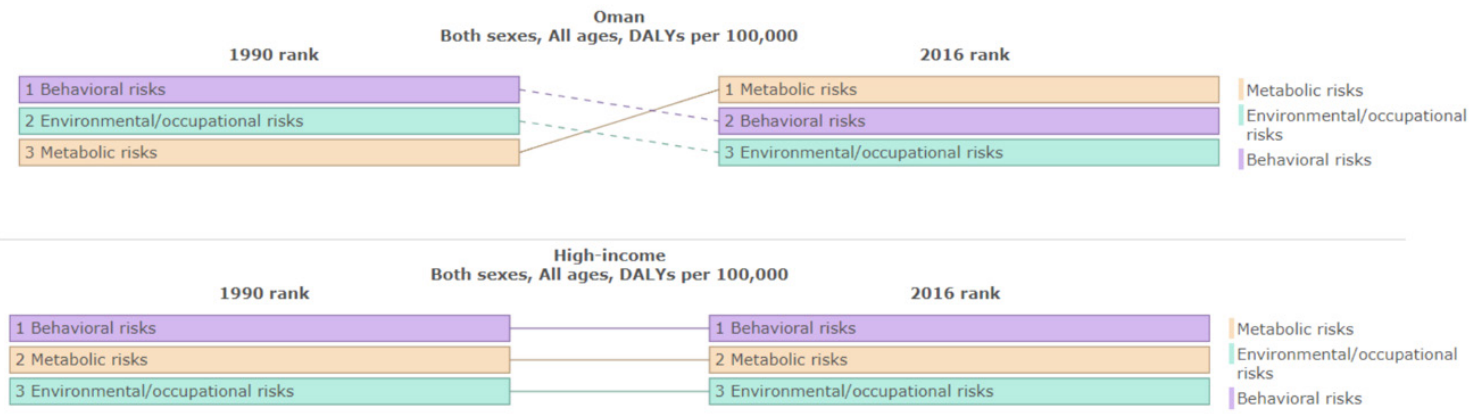

Fig. 3 The risk factors that drive the most disease burden (DALYs) in 0man.

Each risk factors were discussed separately with listing of the most urgent specific research questions. Example of a table constructed for each risk factor can be seen in Table (1).

\section{Top Diseases List}

List of 30 diseases where ranked based on DALYs estimates as follow in Figs 4 and 5:

Each Disease were discussed separately with listing of the most urgent specific research questions. Example of a table constructed for each disease can be seen in Table 1 .

\section{Health System Research}

For the purposes of this exercise, which has been undertaken with limited time and resources, we have categorized the research agenda in HSR into: ${ }^{18}$

\section{1-Functions:}

We have identified what appear to be the key characteristics and core indicators of a well performed health system as recommended by WHO in each building block. ${ }^{19}$ List can be seen in appendix (1)

\section{2-Outcomes:}

A number of principles can inform priority setting in HSR in relation to the outcome. Firstly, there needs to be clear evidence that the problem related to health systems is preventing attainment of the SDGs and that this problem is potentially tractable if addressed by new knowledge from research. Thus, we need to distinguish between the need for better research evidence and solutions that do not require new knowledge, e.g.. an absolute lack of resources that prevents delivery of basic services. Research can however contribute to problem-solving in resource poor environments by, for example, leading to more appropriate policies for financing of health systems, for prioritizing the use of resources and developing an appropriate workforce. ${ }^{20}$ Secondly, it is important to understand the degree to which methodological development is necessary in order to tackle a given research problem and finally to understand how new research can bridge gaps in the existing research portfolio.

Resources used in this exercise of prioritizing health research agenda in HSR: To accomplish this exercise, we have built on work undertaken by the WHO Commission on Macroeconomics and Health that developed a taxonomy of constraints to achieving the SDGs. We have also considered the health policy and systems research key characteristics of health system building blocks derived from WHO publications. We have also taken into account priorities suggested by researchers. Finally, we have taken into account experience from high income countries of research on Service Delivery
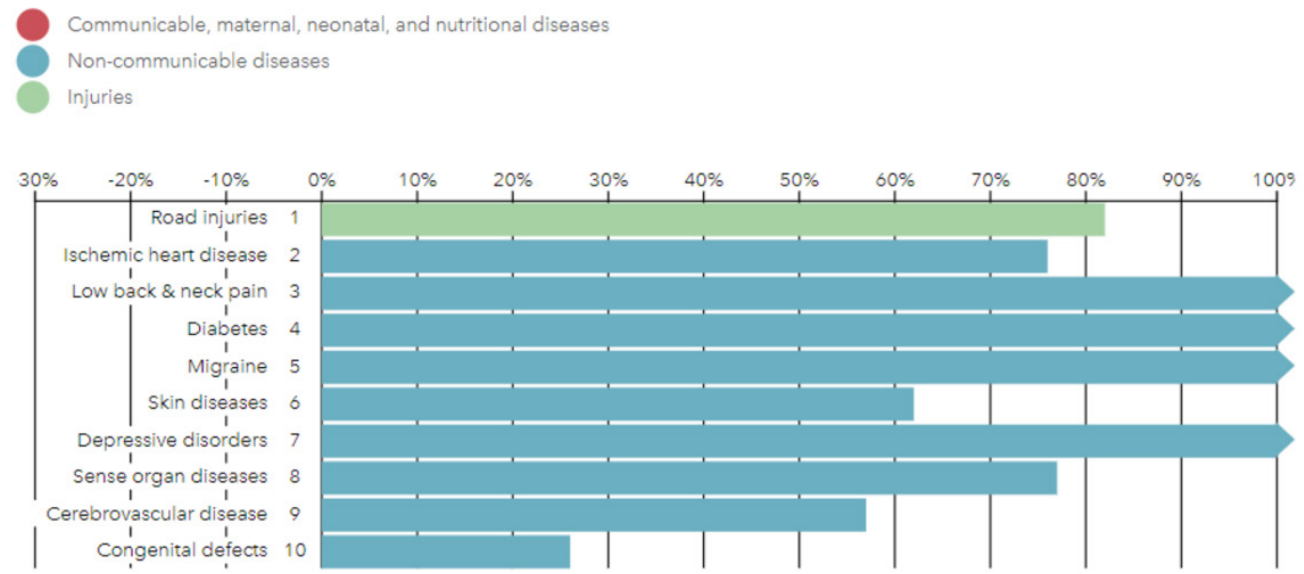

Top 10 causes of disability-adjusted life years (DALYs) in 2016 and percent change, 2005-2016, all ages, number

Fig. 4 List of top 10 diseases where ranked based on DALYs estimates. 


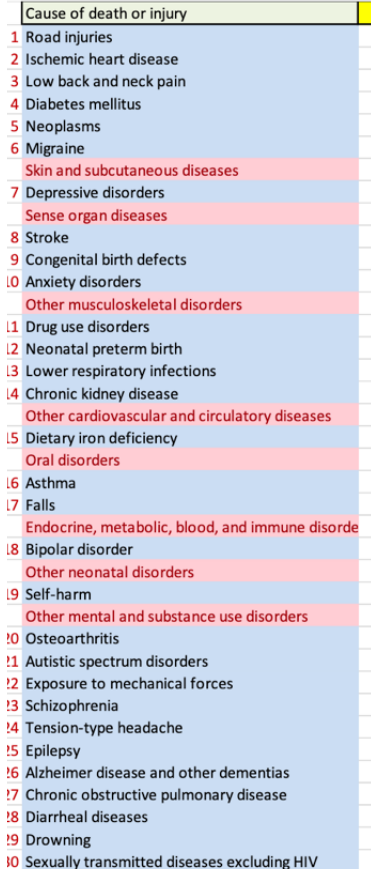

\begin{tabular}{|c|}
\hline DALY \\
\hline $12.73 \%$ \\
\hline $8.85 \%$ \\
\hline $6.16 \%$ \\
\hline $5.98 \%$ \\
\hline $4.49 \%$ \\
\hline $4.08 \%$ \\
\hline $3.61 \%$ \\
\hline $3.35 \%$ \\
\hline $2.85 \%$ \\
\hline $2.63 \%$ \\
\hline $2.61 \%$ \\
\hline $2.42 \%$ \\
\hline $2.36 \%$ \\
\hline $2.13 \%$ \\
\hline $1.78 \%$ \\
\hline $1.68 \%$ \\
$1.55 \%$ \\
\hline $1.33 \%$ \\
\hline $1.30 \%$ \\
\hline $1.19 \%$ \\
\hline $1.18 \%$ \\
\hline $1.16 \%$ \\
\hline $1.04 \%$ \\
\hline $0.95 \%$ \\
\hline $0.90 \%$ \\
\hline $0.87 \%$ \\
$0.87 \%$ \\
\hline $0.83 \%$ \\
\hline $0.81 \%$ \\
\hline $0.78 \%$ \\
$0.77 \%$ \\
$0.76 \%$ \\
\hline $0.76 \%$ \\
\hline $0.73 \%$ \\
\hline $0.70 \%$ \\
$0.65 \%$ \\
$0.61 \%$ \\
$0.59 \%$ \\
\hline
\end{tabular}

\begin{tabular}{l} 
Cause of death or injury \\
3 Low back and neck pain \\
5 Migraine \\
Skin and subcutaneous diseases \\
7 Depressive dissorders \\
4 Diabetes mellitus \\
Sense organ diseases \\
10 Anxiety disorders \\
Other musculoskeletal disorders \\
11 Drug use disorders \\
15 Dietary iron deficiency \\
Oral disorders \\
16 Asthma \\
18 Bipolar disorder \\
1 Road injuries \\
Other mental and substance use disorders \\
20 Osteoarthritis \\
21 Autistic spectrum disorders \\
9 Congenital birth defects \\
17 Falls \\
23 Schizophrenia \\
24 Tension-type headache \\
14 Chronic kidney disease \\
30 Sexually transmitted diseases excluding HIV \\
28 Diarrheal diseases \\
25 Epilepsy \\
Other cardiovascular and circulatory diseases \\
Gynecological diseases \\
Other unintentional injuries \\
2 Ischemic heart disease \\
Alcohol use disorders \\
Upper respiratory infections \\
12 Neonatal preterm birth \\
27 Chronic obstructive pulmonary disease \\
Conduct disorder \\
Urinary diseases and male infertility \\
8 Stroke \\
Hemoglobinopathies and hemolytic anemias \\
22 Exposure to mechanical forces \\
\hline
\end{tabular}

\begin{tabular}{|l|r|}
\hline Cause of death or injury & Deaths \\
\hline 2 Ischemic heart disease & $26.30 \%$ \\
\hline 1 Road injuries & $16.86 \%$ \\
\hline 4 Diabetes mellitus & $8.23 \%$ \\
\hline 8 Stroke & $6.04 \%$ \\
\hline 3 Lower respiratory infections & $3.80 \%$ \\
\hline 26 Alzheimer disease and other dementias & $3.52 \%$ \\
14 Chronic kiddey disease & $2.44 \%$ \\
\hline Other cardiovascular and circulatory diseases & $1.82 \%$ \\
\hline Congenital birth defects & $1.26 \%$ \\
\hline Endocrine, metabolic, blood, and immune disorders & $1.48 \%$ \\
\hline Tracheal, bronchus, and lung cancer & $1.38 \%$ \\
\hline S Self-harm & $1.20 \%$ \\
\hline 2 Neonatal preterm birth & $1.13 \%$ \\
\hline 7 Chronic obstructive pulmonary disease & $1.10 \%$ \\
\hline Colon and rectum cancer & $0.91 \%$ \\
\hline Other neoplasms & $0.87 \%$ \\
\hline Liver cancer & $0.83 \%$ \\
\hline Leukemia & $0.77 \%$ \\
\hline 29 Drowning & $0.77 \%$ \\
\hline Breast cancer & $0.73 \%$ \\
\hline Other neonatal disorders & $0.72 \%$ \\
\hline 2 Exposure to mechanical forces & $0.71 \%$ \\
\hline Non-Hodgkin lymphoma & $0.71 \%$ \\
\hline Stomach cancer & $0.70 \%$ \\
\hline 7 Falls & $0.69 \%$ \\
\hline Cirrhosis and other chronic liver diseases due to hepa & $0.61 \%$ \\
\hline Other unspecified infectious diseases & $0.60 \%$ \\
\hline Cirrhosis and other chronic liver diseases due to hepa & $0.55 \%$ \\
\hline Cardiomyopathy and myocarditis & $0.53 \%$ \\
\hline Brain and nervous system cancer & $0.51 \%$ \\
\hline Encephalitis & $0.51 \%$ \\
\hline Pancreatic cancer & $0.51 \%$ \\
\hline Skin and subcutaneous diseases & $0.46 \%$ \\
\hline Hypertensive heart disease & $0.45 \%$ \\
\hline Prostate cancer & $0.42 \%$ \\
\hline Endocarditis & $0.35 \%$ \\
\hline Atrial fibrillation and flutter & $0.34 \%$ \\
\hline Asthma & $0.33 \%$ \\
\hline
\end{tabular}

Fig. 5 List of top 30 diseases by DALY, YLD, YLL. The red highlighted topics are removed from the main list due to lack of specifications and wide range of diseases included in each category with each single disease might contribute less to overall DALY.

and Organization. We have taken a broad view of HSR and have included within it, issues of management, organization and delivery of health services and research relevant to the development and implementation of policy related to health systems. List of the topics related to the outcome can be found in Appendix 2.

\section{Feasibility Analysis}

Taking into consideration the subnational differences in each perspective of the feasibility assessment, the feasibility assessment done for each governorate separately. So, there will be common national health research priorities but different

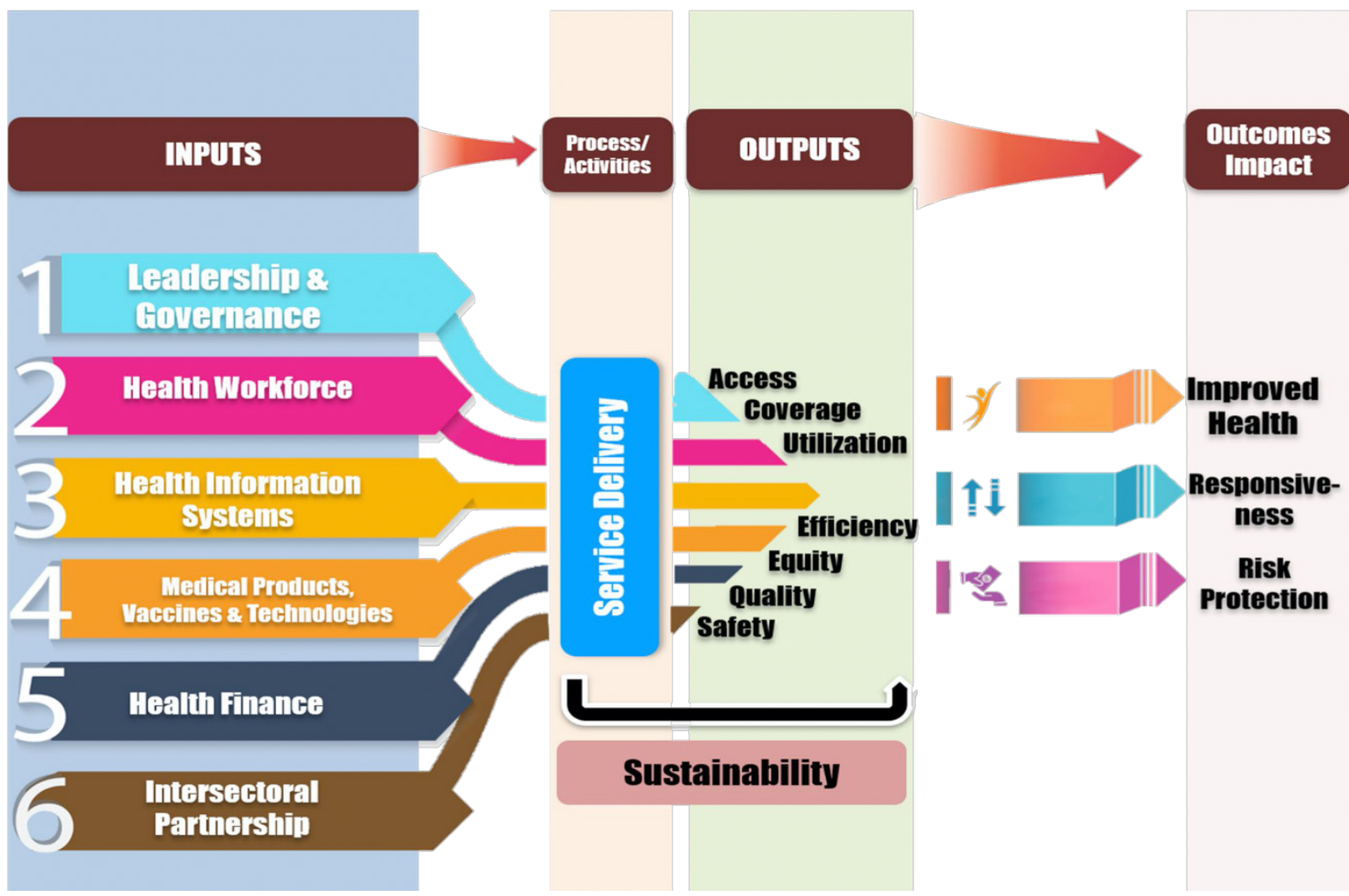

Fig. 6 The complex integration between the inputs, process and output within the Health System. 
specific research questions for each health problem or issue. Table 1 is showing how the diseases or risk factor was integrated with the research questions and feasibility perspectives.

\section{Discussion}

There are number of comprehensive processes for health research priority setting. Consensus on a gold-standard or best practice for health research prioritization thus seems difficult to achieve and is, more importantly, not an appropriate response. However, a nine common themes of good practice for health research prioritization processes have been followed by the Centre of Studies and Research in Oman based on COHRED recommendation to provide assistance for planning a high-quality health research priority setting exercise at national and sub national levels which are ;assessing the contextual factors, choosing the approach, assess the inclusiveness, Identify the information, choosing the criteria, adapt methods, evaluation, transparency and planning for implementation .

Few countries in the world followed the above-mentioned comprehensive process of prioritization as it is a challenging process to follow. ${ }^{6}$ Based on the available resources in Oman, all themes were taken into account and the results were shaped into a 15 years plan. Dissemination of the results were satisfactory at all subnational levels.

Focus groups to continually work on the proposed topics from each subnational level will be managed through a website that also will help in bringing junior researchers and seniors in one channel and for united purposes.

Limitations in each project are inevitable but bringing the maximum quality of the output was the ultimate aim of the exercise of health research priority setting in Oman .Resources were always not enough but getting experts opinions was the only way to fill some gaps in the knowledge needed to continue the exercise. Adherence to the plan will be a challenge that we are planning to compete by regular follow-up and updating.

\section{Conclusion}

In resource rich settings, a high proportion of available research resources go to investigator-driven initiatives, but in limited resource settings, there is an expectation that research must respond more directly to community health needs, and therefore be conducted according to recognized priorities. Prioritization mechanisms are necessary to facilitate the current demand for increased harmonization of health research to meet health services needs particularly in combination with translating the results into actions that improve overall population health. Due to the immense importance of health research in improving the health system and health services, the decision to prioritize health research was the first step in the direction to enhance health research and thereby enhance health service in Oman by the Centre of Studies \& Research. This intervention will lead the health system to achieve better planning for effective utilization of available resources.

\section{Competing interests}

The authors declare that they have no competing interests.

\section{References}

1. McGregor S, Henderson KJ, Kaldor JM. How are health research priorities set in low- and middle-income countries? A systematic review of published reports. PloS one, 2014;9:e108787.

2. Al-Mawali A. Non-Communicable Diseases: Shining a Light on Cardiovascular Disease, Oman's Biggest Killer. Oman Med J. 2015 Jul;30(4):227-8. doi: 10.5001/omj.2015.47. PMID: 26366254; PMCID: PMC4561645

3. Angelis A, Kanavos P, Montibeller G. Resource allocation and priority setting in health care: A multi- criteria decision analysis problem of value? Global Policy, 2017:8:76-83

4. Council on Health Research for Development (COHRED). Essential National Health Research and Priority Setting: Lessons Learned. 1997

5. Dolan P, Tsuchiya A. Health priorities and public preferences: the relative importance of past health experience and future health prospects. J Health Econ, 2005;24:703-714.

6. Viergever RF, Olifson S, Ghaffar A, Terry RF. A checklist for health research priority setting: nine common themes of good practice. Health Res Policy Syst, 2010;8:36.

7. Ministry of Health Oman. Ninth Five Year-Plan for Health Development. 2015

8. Ministry of Health Oman. Annual Health Report 2016

9. Fryatt R, Mills A, Nordstrom A. Financing of health systems to achieve the health Millennium Development Goals in low-income countries. Lancet, 2010;375:419-426.

10. Deng G, Weber W, Sood A, Kemper KJ. Research on integrative healthcare: context and priorities. Explore: J Sci Heal, 2010;6: 143-158.

11. Al-Mawali, A, IA John, DP Avinash. Fraud and misconduct in clinical research: A step to improve ethical practice in research. J Contemp Med Sci, 2010;4(3 Sep. 2018). ISSN 2413-0516. Available at: <http://www.jocms.org/ index.php/jcms/article/view/449>. Date accessed: 29 june 2020

12. Ghaffar A, Collins T, Matlin S, Olifson S. The 3D Combined Approach Matrix: An improved tool for setting priorities in research for health. Geneva: Global Forum for Health Research. 2009.

13. Okello D, Chongtrakul P. A manual for research priority setting using the ENHR strategy. COHRED. 2000

14. El Lawindi MI, Galal YS, Khairy WA. Health research and millennium development goals: identifying the gap from public health perspective. Global J Health Sci, 2016:8:1.

15. Institute for Health Metrics and Evaluation (IHME). Global Burden of Disease Study 2016 (GBD 2016). 2016

16. World Health Organization. The Global Burden of Disease concept. (Available from http://www.who.int/quantifying_ehimpacts/publications/ en/9241546204chap3.pdf). Accessed on July 1, 2018.

17. Mitchell S, Shaw D. The worldwide epidemic of female obesity. Best Pract Res Clin Obstet Gynaecol, 2015;29:289-99.

18. World Health Organization. Framework for developing a health systems research agenda. (Available from http://www.who.int/rpc/meetings/ Framework_for_developing_a_health_systems_research_agenda.pdf). Accessed on July 3, 2018.

19. Viergever RF, Terry R, Matsoso M. Health research prioritization at WHO: an overview of methodology and high-level analysis of WHO led health research priority setting exercises. Geneva: World Health Organization. 2010

20. Kutzin J. A descriptive framework for country-level analysis of health care financing arrangements. Health Policy, 2001;56:171-204.

21. Montorzi G, De Haan S, IJsselmuiden C. Priority Setting for Research for Health: a management process for countries. Council on Health Research for Development (COHRED). 2010

22. Whear R, Thompson-Coon J, Boddy K, et al. Establishing local priorities for a health research agenda. Health Expect, 2015;18:8-21.

23. National Association of County and City Health Officials (NACCHO). Tip Sheet: Prioritizing Issues in a Community Health Improvement Process. Community Health Assessments and Community Health Improvement Plans for Accreditation Preparation Demonstration Project. 2010.

24. Organization WH. A Research policy agenda for science and technology to support global health development: a synopsis. Geneva: World Health Organization. 1997

25. Al Mawali AHN, Al Qasmi AM, Al Sabahi SMS, Idikula J, Elaty MAA, Morsi M, Al Hinai AT. Oman vision 2050 for health research: A strategic plan for the future based on the past and present experience. Oman Med J. 2017 Mar;32(2):86-96. doi: 10.5001/omj.2017.18. PMID: 28439378; PMCID: PMC5397084. 
26. Oman Health vision 2050, published by undersecretary of Planning, Ministry of Health, Sultanate of Oman. 2015

27. Ranson MK, Bennett SC. Priority setting and health policy and systems research. Health Res. Policy Syst, 2009:7: 27

28. Garcia AB, Cassiani SHDB, Reveiz L. A systematic review of nursing research priorities on health system and services in the Americas. Rev. Panam. de Salud Públ., 2015;37:162-171.

29. Cooke J, Ariss S, Smith C, Read J. On-going collaborative priority-setting for research activity: a method of capacity building to reduce the researchpractice translational gap. Health Res Policy Syst, 2015;13:25
30. Madon T, Hofman KJ, Kupfer L, Glass RI. Implementation science. American Association for the Advancement of Science. 2007.

31. World Health Organization. A prioritized research agenda for prevention and control of noncommunicable diseases. 2011

32. Mirzoev T, Kane S. What is health systems responsiveness? Review of existing knowledge and proposed conceptual framework. BMJ Global Health, 2017;2:e000486.

33. Remme JH, Adam T, Becerra-Posada F, et al. Defining research to improve health systems. PLoS Med, 2010;7:e1001000.

This work is licensed under a Creative Commons Attribution-NonCommercial 3.0 Unported License which allows users to read, copy, distribute and make derivative works for non-commercial purposes from the material, as long as the author of the original work is cited properly. 